\title{
Integrability, Stäckel spaces, and rational potentials
}

\author{
E. G. Kalnins \\ Department of Mathematics and Statistics, University of Waikato, Hamilton, New Zealand
}

S. Benenti

Istituto Di Fisica Matematica, Universita Di Torino, Torino, Italy

Willard Miller, Jr.

School of Mathematics and Institute for Mathematics and its Applications,

University of Minnesota, Minneapolis, Minnesota 55455

(Received 27 November 1995; accepted for publication 28 October 1996)

For a variety of classical mechanical systems embeddable into flat space with Cartesian coordinates $\left\{x_{i}\right\}$ and for which the Hamilton-Jacobi equation can be solved via separation of variables in a particular curvalinear system $\left\{u_{j}\right\}$, we answer the following question. When is the separable potential function $v$ expressible as a polynomial (or as a rational function) in the defining coordinates $\left\{x_{i}\right\}$ ? Many examples are given. () 1997 American Institute of Physics.

[S0022-2488(97)01905-1]

\section{INTRODUCTION}

In recent years there has been renewed interest in the notion of integrability as it applies to the solution of mechanical systems. Many examples of such systems are known. The crucial requirement for the notion of integrability to be valid is the existence of a suitable number of constants of the motion. This guarantees in principle that the corresponding mathematical problem can be solved. One of the classical ways of solving some integrable mechanical systems is using the method of separation of variables. Commonly quoted integrable systems are the so-called Neumann system of a particle moving on a sphere under the influence of the anisotropic harmonic potential and also the problem of geodesic flow on an ellipsoid. ${ }^{1-4}$ The problem we consider here is, generally, how to classify systematically those classical mechanical systems that are of the form

$$
H=\sum_{i, j=1}^{n} g^{i j} p_{i} p_{j}+V\left(x^{1}, \ldots, x^{n}\right) .
$$

via separation of variables techniques. Here the metric $g^{i j}$ corresponds to some Riemannian space that is embedded into flat space and the $x^{i}$ are Cartesian coordinates in that space. Specifically, we will determine the possible separable potentials $V$ which are polynomials or rational functions of the Cartesian coordinates. In particular we consider the case of spaces of constant curvature, i.e., Euclidean space, the positive definite hyperboloid and the sphere. Also considered here are complex versions of these spaces. For the real spaces we have mentioned, all the separable coordinate systems are known. ${ }^{5,6}$ The requirement that the potential provide a separation of variables is also known (e.g., Ref. 7). Indeed if the coordinates $y^{i}$ provide a separation of variables via some transformation $y^{i}=y^{i}\left(x^{1}, \ldots, x^{n}\right), i=1, \ldots, n$, the only nonzero elements of the contravariant tensor

$$
\bar{g}^{i j}=\sum_{p, q=1}^{n} g^{p q} \frac{\partial y^{i}}{\partial x^{p}} \frac{\partial y^{j}}{\partial x^{q}}
$$

$\operatorname{are}^{8-15}$ 


$$
\begin{aligned}
& g^{i j}=\delta^{i j}\left[S^{i 1} / S\right], \quad i, j=1, \ldots, n_{1}, \\
& g^{r \alpha}=\left[S^{r 1} / S\right] f_{r}\left(y^{r}\right), \quad r=n_{1}+, \ldots, n_{1}+n_{2}+2, \quad \alpha=n_{1}+n_{2}+2, \ldots, n_{1}+n_{2}+n_{3}+2, \\
& g^{\alpha \beta}=\left[\sum_{k=1}^{n_{1}+n_{2}} h_{k}^{\alpha \beta}\left(y^{k}\right)\left(S^{k 1} / S\right)\right], \quad \alpha, \beta=n_{1}+n_{2}+2, \ldots, n_{1}+n_{2}+n_{3}+2 .
\end{aligned}
$$

Here $n_{1}+n_{2}+n_{3}=n$, and there exists a Stäckel matrix $\bar{S}=\left(\bar{S}_{i j}\left(y^{i}\right)\right)_{n \times n}$ such that $S=\operatorname{det} \bar{S}$ and $S^{i j}$ is the $i j$ cofactor of $\bar{S}$. The requirement that the potentials separate in these coordinates is that ${ }^{7}$

$$
V\left(y^{1}, \ldots, y^{n}\right)=\sum_{k=1}^{n_{1}+n_{2}} v\left(y^{k}\right) \frac{S^{k 1}}{S} .
$$

When this is the case $V$ is said to be a Stäckel multiplier in the coordinate system $y^{i}$. Coordinates of the type $y^{i}, i=1, \ldots, n_{1}$, are called Stäckel coordinates. Coordinates of type $y^{r}, r=n_{1}$ $+1, \ldots, n_{1}+n_{2}+2$, are called first-order coordinates. Coordinates of type $y^{\alpha}, \alpha=n_{1}+n_{2}$ $+2, \ldots, n_{1}+n_{2}+n_{3}+2$, are called ignorable variables. We will restrict ourselves here to coordinate systems such that $\vec{g}^{i j}$ is a diagonal metric. This is both necessary and sufficient for the case of separation of variables for real spaces of constant curvature. ${ }^{6}$

In the case of real Euclidean space let us consider the generic elliptical coordinates $\left\{u_{i}\right\}$ defined by

$$
x_{i}^{2}=c^{2} \Pi_{j=1}^{n}\left(u_{j}-e_{i}\right) / \Pi_{k \neq i}\left(e_{k}-e_{i}\right), \quad i, j, k=1, \ldots, n,
$$

and $e_{i} \neq e_{k}$ for $i \neq k$. For elliptical coordinates the new variables are subject to the restrictions $e_{1}<u_{1}<e_{2}<u_{2}<\cdots<e_{n}<u_{n}$. The general form for the Stäckel multiplier associated with these coordinates can be readily calculated. This may be deduced from the infinitesimal distance

$$
d s^{2}=\frac{c^{2}}{4} \sum_{k=1}^{n} \frac{\Pi_{m \neq i}\left(u_{m}-u_{i}\right)}{\Pi_{l=1}^{n}\left(u_{k}-e_{l}\right)} d u_{k}^{2}
$$

Indeed the form of such a Stäckel multiplier must be

$$
V\left(u_{1}, \ldots, u_{n}\right)=\sum_{i=1}^{n} \frac{v_{i}\left(u_{i}\right)}{\Pi_{m \neq i}\left(u_{m}-u_{i}\right)} .
$$

In this sense this problem has been solved. However, we seek a solution to a more restricted problem here. Indeed we answer the following two questions.

(1) When is the Stäckel multiplier $V\left(u_{1}, \ldots, u_{n}\right)$ expressible as a polynomial in the defining coordinates $x_{i}$ ?

(2) When is the multiplier in the form of a rational function of the Cartesian coordinates $x_{i}$ ?

These are two important classes of potentials and a number of physically interesting cases are known. Indeed Eisenhart has shown that in an orthogonal separable system $y^{i}$ a potential is in the form of a Stäckel multiplier if and only if

$$
g^{i i} g^{j j} \frac{\partial^{2} V}{\partial u_{i} \partial u_{j}}-g^{i i} \frac{\partial g^{j j}}{\partial u_{i}} \frac{\partial V}{\partial u_{j}}-g^{j j} \frac{\partial g^{i i}}{\partial u_{j}} \frac{\partial V}{\partial u_{i}}=0
$$

for $i \neq j .{ }^{9}$ However, Benenti ${ }^{16}$ has proved that (expressed in Cartesian coordinates) these conditions for elliptical coordinates are equivalent to 


$$
\left(e_{i}-e_{j}\right) \frac{\partial^{2} V}{\partial x_{i} \partial x_{j}}+\left(x_{i} \frac{\partial}{\partial x_{j}}-x_{j} \frac{\partial}{\partial x_{i}}\right)\left(2 V+\sum_{k=1}^{n} x_{k} \frac{\partial V}{\partial x_{k}}\right)=0
$$

for $i \neq j$. Thus for elliptical coordinates, the solutions to questions (1) and (2) are just the polynomial or rational solutions to (1.6). We will give a different and more general solution to these questions.

We first look at the problem of polynomial potentials. The crucial observation is the following. Let $V\left(x_{1}, \ldots, x_{n}\right)$ be a polynomial solution of (1.6). When expressed as a function of the $u_{i}$ coordinates via (1.3), $V$ should be symmetric. Therefore when expressed in the form (1.4) each of the functions $v_{i}\left(u_{i}\right)=v\left(u_{i}\right)$. We can deduce $v\left(u_{i}\right)$ if $V$ is of polynomial form. Indeed if we put $u_{i}=a_{i}$ for $i \neq 1$ such that $a_{k} \neq e_{l}$ for all $k$ and $l$, then we see that $v(u)$ should have the form $v(u)=R(u) \sqrt{u-e_{1}}+S(u)$ where $R$ and $S$ are rational functions of $u$. Doing a similar calculation with $e_{1}$ replaced by $e_{2}$ we must then have

$$
R(u) \sqrt{u-e_{1}}+S(u)=R^{\prime}(u) \sqrt{u-e_{2}}+S^{\prime}(u) .
$$

This can only hold if $R(u)=R^{\prime}(u)=0$ and $S(u)=S^{\prime}(u)$. Before proceeding further we note the following.

Lemma. Let $S_{i}\left[u_{1}, \ldots, u_{n}\right]$ be the symmetric polynomial defined by

$$
S_{i}\left[u_{1}, \ldots, u_{n}\right]=\frac{1}{i !} \sum_{j_{1}, \ldots, j_{i} \neq} u_{j_{1}}, \ldots, u_{j_{i}}, \quad i=1, \ldots, n,
$$

and $S_{0}\left[u_{1}, \ldots, u_{n}\right]=0$. Similarly let $E_{i}\left[e_{1}, \ldots, e_{n}\right]=S_{i}\left[e_{1}, \ldots, e_{n}\right]$. Then the symmetric functions $S_{i}\left[u_{1}, \ldots, u_{n}\right]$ are expressible in terms of the Cartesian coordinates via

$$
S_{l}\left[u_{1}, \ldots, u_{n}\right]=E_{l}\left[e_{1}, \ldots, e_{n}\right]+\sum_{k=1}^{n} \frac{x_{k}^{2}}{c^{2}} E_{l-1}\left[e_{1}, \ldots, e_{k-1}, e_{k+1}, \ldots, e_{n}\right], \quad l=1, \ldots, n
$$

This follows readily from the identity

$$
\sum_{k=1}^{n} \frac{x_{k}^{2}}{\left(u-e_{k}\right)}-c^{2}=-c^{2} \frac{\Pi_{k=1}^{n}\left(u-u_{k}\right)}{\Pi_{j=1}^{n}\left(u-e_{j}\right)}
$$

Multiplying this expression by $\Pi_{j=1}^{n}\left(u-e_{j}\right)$ we easily deduce the identity (1.8). The functions $S M_{p}\left[u_{1}, \ldots, u_{n}\right]=\sum_{i=1}^{n} u_{i}^{p} / \Pi_{j \neq i}\left(u_{i}-u_{j}\right)$ for $p$ an integer can be expressed in terms of Cartesian coordinates via the recurrence relation

$$
S M_{q}=\sum_{j=1}^{n}(-1)^{j+1} S_{j}\left[u_{1}, \ldots, u_{n}\right] S M_{q-j},
$$

for $q \geqslant n$, where $S M_{n-1}=1$ and $S M_{p}=0$ for $0 \leqslant p<n-1$.

Consequently, if $v(u)$ is a polynomial in $u$, the corresponding potential is a polynomial in the Cartesian coordinates. We can conclude that all contributions for which $v(u)=u^{p}$ give a basis for all Stäckel multipliers which are polynomial in the Cartesian coordinates $x_{k}$. The second type of contribution viz. $1 /(u-a)^{q}$, can be readily expressed in terms of Cartesian coordinates. Indeed we need only consider the special case $a=0$, as the general case can be obtained by translating all the $u_{i}$ variables. We observe that $S M_{-p}\left[u_{1}, \ldots, u_{n}\right]=\left(\Pi_{i=1}^{n} v_{i}\right) S M_{p+n-2}\left[v_{1}, \ldots, v_{n}\right]$ where $v_{i}$ $=1 / u_{i}$. Then using the result $S_{q}\left[v_{1}, \ldots, v_{n}\right]=S_{n-q}\left[u_{1}, \ldots, u_{n}\right] /\left(\Pi_{i=1}^{n} u_{i}\right)$ we can always evaluate 
$S M_{-p}\left[u_{1}, \ldots, u_{n}\right]$. The analysis is now complete. The basic building blocks for potentials which are rational functions of the Cartesian coordinates are the Stäckel multipliers $S M_{p}\left[u_{1}, \ldots, u_{n}\right]$ where $p=0, \pm 1, \pm 2, \ldots$.

For real Euclidean spaces there is one other generic coordinate system, paraboloidal coordinates. These coordinates are given by

$$
\begin{gathered}
x_{1}=\left(\frac{c}{2}\right)\left(-\sum_{j=1}^{n} u_{j}+\sum_{k=1}^{n-1} e_{k}\right), \\
x_{i}^{2}=c^{2} \frac{\prod_{j=1}^{n}\left(u_{j}-e_{i-1}\right)}{\prod_{k \neq i-1}\left(e_{k}-e_{i-1}\right)}, \quad i, k=2, \ldots, n, \quad j=1, \ldots, n,
\end{gathered}
$$

where $e_{1}<u_{1}<e_{2}<u_{2}<e_{3}<\cdots<u_{n-1}<e_{n}<u_{n}$. The corresponding infinitesmal distance is

$$
d s^{2}=\frac{c^{2}}{4} \sum_{k=1}^{n} \frac{\Pi_{m \neq i}\left(u_{m}-u_{i}\right)}{\Pi_{l=1}^{n-1}\left(u_{k}-e_{l}\right)} d u_{k}^{2}
$$

and the symmetrical functions $S_{i}\left[u_{1}, \ldots, u_{n}\right]$ are given by the expressions

$$
\begin{gathered}
c^{2} S_{1}\left[u_{1}, \ldots, u_{n}\right]=E_{1}\left[e_{2}, \ldots, e_{n}\right]-2 x_{1}, \\
c^{2} S_{l}\left[u_{1}, \ldots, u_{n}\right]=E_{l}\left[e_{2}, \ldots, e_{n}\right]-2 x_{1} E_{l-}\left[e_{2}, \ldots, e_{n}\right]-\sum_{k=2}^{n} x_{k}^{2} E_{l-2}\left[e_{2}, e_{k-1}, e_{k+1}, \ldots, e_{n}\right], \\
l=2, \ldots, n-1, \\
c^{2} S_{n}\left[u_{1}, \ldots, u_{n}\right]=-2 x_{1} E_{n-1}\left[e_{2}, \ldots, e_{n}\right]-\sum_{k=2}^{n} x_{k}^{2} E_{n-2}\left[e_{2}, e_{k-1}, e_{k+1} \ldots, e_{n}\right] .
\end{gathered}
$$

This follows from the identity

$$
-2 c x_{1}-c^{2} u+\sum_{k=2}^{n} \frac{x_{k}^{2}}{\left(u-e_{k}\right)}=c^{2} \frac{\Pi_{k=1}^{n}\left(u-u_{k}\right)}{\Pi_{j=2}^{n}\left(u-e_{j}\right)} .
$$

To establish which potentials are Stäckel multipliers for paraboloidal coordinates and which are rational functions of the Cartesian coordinates we must consider two cases.

(1) $n>2$. In this case we can use essentially the previous argument for elliptical coordinates in

$E_{n}$. Then, as expected, linear combinations of $v_{i}(u)=v(u)=(u-a)^{p}, p=0, \pm 1, \pm 2, \ldots$, form a basis for all Stäckel multipliers that are rational functions of Cartesian coordinates.

(2) $n=2$. In this case we cannot use the previous argument, as there is only one $e_{k}$ occurring, viz.

$e_{2}$, which we can take to be 0 . We can only deduce that $v(u)=R(u) u^{1 / 2}+S(u)$ where $R$ and $S$ are rational functions. If $R(u)=0$, then our result follows as for $n>2$. If $R(u) \neq 0$, then we change to the new variable $w=u^{1 / 2}$ and discard the function $S(u)$. The corresponding Stäckel multiplier has the form

$$
v\left(w_{1}, w_{2}\right)=\frac{T\left(w_{1}\right)-T\left(w_{2}\right)}{w_{1}^{2}-w_{2}^{2}}
$$

where $T(w)$ is a rational function of $w$ of the form $R\left(w^{2}\right) w$. If we perform a partial fraction decomposition of $R(u)$ with respect to $u$, then the function $T(w)$ typically has terms of the type/1 
which are monomials $w^{p}$ and terms of type/2 which are of the form $w /\left(w^{2}-a^{2}\right)^{p}$. In the case of monomials, if $p$ is even, then the corresponding contribution to the Stäckel multiplier is a polynomial in the Cartesian coordinates $x_{k}$. If $p$ is odd, then the corresponding contribution can be written as an algebraic function of the symmetric functions $S_{i}\left[w_{1}, w_{2}\right]$. Furthermore, these symmetric functions cannot be expressed as rational functions of $x_{k}$. Indeed they are determined by algebraic relations. For example, in the case of

$$
S_{1 / 2}\left[u_{1}, u_{2}\right]=S_{1}\left[w_{1}, w_{2}\right]=w_{1}+w_{2}=u_{1}^{1 / 2}+u_{2}^{1 / 2},
$$

the relation between $S_{1 / 2}$ and the symmetric polynomials $S_{i}=S_{i}\left[u_{1} u_{2}\right]$ is given by

$$
\left[S_{1 / 2}^{2}-S_{1}\right]^{2}=4 S_{2}^{2} \text {. }
$$

In a similar way we could argue that contributing terms to $v\left(w_{1}, w_{2}\right)$ of the form $1 /(w$ $-a)^{p}$ cannot contribute rational functions of $x_{k}$ to the expression for a separable potential. We are thus left with the possibility that $v(u)$ must be a rational function of $u$. Consequently $v(u)$ admits a partial fraction decomposition with typical terms of the form $u^{p}$ or $1 /(u-a)^{q}$ for $p$ and $q$ positive integers.

In the following sections we will answer questions (1) and (2) for a variety of classical mechanical systems embeddable into flat space with Cartesian coordinates $\left\{x_{i}\right\}$ and for which the Hamilton-Jacobi equation can be solved via separation of variables in a particular curvalinear system $\left\{u_{j}\right\}$. Many examples are given in the Appendix.

In summary, the conclusions of Sec. I are as follows. It is known that separable coordinates in $n$-dimensional Euclidean space are constructed from generic separable coordinate systems viz. elliptical coordinates and paraboloidal coordinates. ${ }^{6}$ For these coordinate systems we have asked what potentials rational in the Cartesian coordinates can be found such that solution via the separation of variables ansatz is still valid. We have shown that potentials fulfilling this requirement can be constructed from the symmetric quantities $S_{l}\left[u_{1}, \ldots, u_{n}\right]$ and that these quantities can always be expressed as a rational function of the Cartesian coordinates $x_{k}$ via (1.8). These ideas are extended in subsequent sections to the case of the sphere and the hyperboloid in $n$ dimensions. The result of these investigations gives all potentials which are rational in terms of Cartesian coordinates and have the separability property. In addition we develop these ideas even further to include spaces in which the rational Stäckel form of elliptic coordinates is generalized and show that similar results hold in this case also. This we do via what amounts to flat space embeddings.

\section{ELLIPTIC COORDINATES ON THE $\boldsymbol{n}$ SPHERE}

The application of these ideas to the case of elliptic coordinates on the $n$ sphere proceeds with some modifications. Elliptic coordinates on $S_{n}$ are given by

$$
x_{i}^{2}=\frac{\prod_{j=1}^{n}\left(u_{j}-e_{i}\right)}{\prod_{k \neq i}\left(e_{k}-e_{i}\right)}, \quad i, k=1, \ldots, n+1, \quad j=1, \ldots, n,
$$

subject to $e_{1}<u_{1}<e_{2}<u_{2}<\cdots<e_{n}<u_{n}<e_{n+1}$ and $\sum_{i=1}^{n+1} x_{i}^{2}=1$. A typical form of Stäckel multiplier is

$$
V\left(u_{1}, \ldots, u_{n}\right)=\sum_{i=1}^{n} \frac{v_{i}\left(u_{i}\right)}{\Pi_{m \neq i}\left(u_{m}-u_{i}\right)} .
$$

Again we ask the question which separable potentials can be expressed as rational functions of the projective coordinates $x_{k}$. The expression (1.9) is now replaced by 


$$
\sum_{k=1}^{n+1} \frac{x_{k}^{2}}{u-e_{k}}=\frac{\Pi_{k=1}^{n}\left(u-u_{k}\right)}{\prod_{j=1}^{n+1}\left(u-e_{j}\right)},
$$

from which we deduce that

$$
S_{l}\left[u_{1}, \ldots, u_{n}\right]=\sum_{k=1}^{n+1} x_{k}^{2} E_{l}\left[e_{1}, \ldots, e_{k-1}, e_{k+1}, \ldots, e_{n}\right] .
$$

The argument used for the case of Euclidean elliptic coordinates goes through much as before. Indeed the Stäckel multipliers $S M_{p}\left[u_{1}, \ldots, u_{n}\right]$, with $p=0, \pm 1, \pm 2, \ldots$, form a basis for all such potentials. Among these potentials are several well-known examples of separable coordinate systems on the $n$ sphere. Indeed $V=S_{1}\left[u_{1}, \ldots, u_{n}\right]=\sum_{i=1}^{n} u_{i}$ corresponds to the Neumann potential $\sum_{i=1}^{n+1} e_{i}\left(1-x_{i}^{2}\right){ }^{1,3,7}$ The Stäckel multiplier $1 / x_{k}^{2}$ corresponds to the choice of function $v(u)=1$ $-\sum_{k=1}^{n+1} \Pi_{l \neq k}\left(u-e_{l}\right) /\left(u-e_{k}\right)$. The often quoted Rosochatius potential $V=\sum_{k=1}^{n+1} c_{k} / x_{k}^{2},{ }^{17}$ can then be constructed from the corresponding Stäckel multipliers. For the Garnier system with potential

$$
V\left(x_{1}, \ldots, x_{n}\right)=\left(\sum_{k=1}^{n} x_{k}^{2}\right)^{2}-\left(\sum_{k=1}^{n} E_{1}\left[e_{1}, \ldots, e_{k-1}, e_{k+1}, \ldots, e_{n}\right] x_{k}^{2}\right),
$$

the corresponding function is

$$
v_{k}(u)=u^{n+1}-2 E_{1}\left[e_{1}, \ldots, e_{n}\right] u^{n}+\left(E_{2}\left[e_{1}, \ldots, e_{n}\right]-E_{1}\left[e_{1}, \ldots, e_{n}\right]^{2}\right) u^{n-1}+2 E_{1}\left[e_{1}, \ldots, e_{n}\right]^{2} .
$$

\section{COMPLEX $S_{n}$, COMPLEX $E_{n}$, AND LIMITING REAL CASES}

Having solved the problem of rational potentials for generic coordinates on the $n$ sphere and in Euclidean $n$ space we can now answer the same question for degenerate versions of these coordinates. We make use of well-established limiting procedures. ${ }^{7,18}$ In particular we consider those cases relevant to the real manifolds (1) Euclidean $n$ space $E_{n}$, (2) the $n$ sphere $S_{n}$, and (3) the $n$-dimensional hyperboloid $H_{n}$. This can be best done by first considering complex Euclidean $n$ space and the complex $n$ sphere, and then passing to the real cases. For the complex $n$ sphere let us consider what modifications are necessary for the solution of our problems. The process of taking two roots $e_{i}$ equal best illustrates the general procedure. Indeed if we put

$$
\begin{gathered}
x_{2} \rightarrow \sqrt{a_{2}}\left(x_{1}^{\prime}+\epsilon x_{2}^{\prime}\right), \quad x_{1} \rightarrow \sqrt{a_{1}} x_{1}^{\prime}, \\
x_{j} \rightarrow x_{j}^{\prime}, \quad j=3, \ldots, n,
\end{gathered}
$$

where $a_{1}=-a_{2}=1 / \epsilon$ and $e_{2}=e_{1}+\epsilon$, then the generic elliptic coordinates on the $n$ sphere become

$$
\begin{gathered}
x_{1}^{\prime 2}=\prod_{i=1}^{n}\left(u_{i}-e_{1}\right) / \Pi_{k=3}^{n+1}\left(e_{k}-e_{1}\right), \\
2 x_{1}^{\prime} x_{2}^{\prime}=\left(\frac{\partial}{\partial e_{1}}\right)\left[\prod_{i=1}^{n}\left(u_{i}-e_{1}\right) / \Pi_{k=3}^{n+1}\left(e_{k}-e_{1}\right)\right], \\
x_{j}^{\prime 2}=\prod_{i=1}^{n}\left(u_{i}-e_{j}\right) / \prod_{k \neq j}^{n+1}\left(e_{k}-e_{j}\right), \quad j=3, \ldots, n+1, \\
2 x_{1}^{\prime} x_{2}^{\prime}+\sum_{i=3}^{n+1} x_{i}^{\prime 2}=1 .
\end{gathered}
$$


For this example, the argument used previously in the generic case is readily adapted. Indeed if we take a Stäckel multiplier in the form (1.4), the function $v(u)$ must be a rational function of $u$. Accordingly the basis for the rational Stäckel multipliers in this coordinate system is exactly the same when written in terms of the coordinates $u_{i}$. To express these Stäckel multipliers in terms of the coordinates $x_{i}^{\prime}$ we need only take the appropriate limits in the expressions (1.9). The functions $S_{i}\left[u_{1}, \ldots, u_{n}\right]$ can be obtained from the new form of (1.9), viz.

$$
\frac{2 x_{1} x_{2}}{u-e_{1}}+\frac{x_{1}^{2}}{\left(u-e_{1}\right)^{2}}+\sum_{k=3}^{n+1} \frac{x_{k}^{2}}{u-e_{k}}=\frac{\Pi_{k=1}^{n}\left(u-u_{k}\right)}{\left(u-e_{1}\right)^{2} \Pi_{j=3}^{n+1}\left(u-e_{j}\right)} .
$$

For the explicit form of the functions $S_{l}\left[u_{1}, \ldots, u_{n}\right]$ we obtain

$$
\begin{aligned}
S_{l}\left[u_{1}, \ldots, u_{n}\right]= & 2 x_{1} x_{2} E_{l}\left[e_{1}, e_{3}, \ldots, e_{n+1}\right]+x_{1}^{2} E_{l-1}\left[e_{3}, \ldots, e_{n+1}\right] \\
& +\sum_{k=3}^{n+1} x_{k}^{2} E_{l}\left[e_{1}, e_{1}, \ldots, e_{k-1}, e_{k+1}, \ldots, e_{n+1}\right] .
\end{aligned}
$$

Here we have dropped the primes in the $x_{k}$. We note in particular from (3.3) that $2 x_{1} x_{2}$ $+\sum_{k=3}^{n+1} x_{k}^{2}=1$. For this particular case the Neumann potential becomes

$$
V=2 e_{1}+e_{3}+\cdots+e_{n+1}-\left(x_{1}^{2}+2 e_{1} x_{1} x_{2}+\sum_{k=3}^{n+1} x_{k}^{2}\right)
$$

The most general possibility of this kind occurs when the $e_{i}$ s are equal in groups. Specifically, if we write instead of $x_{i}$ and $e_{k}$ the new variables $x_{j}^{J}$ and $e_{j}^{J}$, where $j=1, \ldots, N_{J}$, and $J$ $=1, \ldots, P$, respectively, the infinitesimal distance is

$$
d s^{2}=\sum_{k=1}^{n} \frac{\Pi_{m \neq i}\left(u_{m}-u_{i}\right)}{\Pi_{j=1}^{N_{J}}\left(u_{k}-e_{j}^{J}\right)} d u_{k}^{2} .
$$

These coordinates can be obtained from the most general elliptic ones by well-defined limiting processes. ${ }^{13}$ Under the transformation

$$
\begin{gathered}
e_{j}^{J} \rightarrow e_{J}+{ }^{J} \epsilon_{j-1}^{1}, \quad j=1, \ldots, N_{J}, \quad J=1, \ldots, P, \\
x_{j}^{J} \rightarrow \sqrt{a_{j}^{J}}\left(x_{1}^{J}+\sum_{i=2}^{N_{J}}{ }^{J} \epsilon_{j+1-i}^{i-1} x_{i}^{J}\right),
\end{gathered}
$$

where

$$
{ }^{J} \epsilon_{j+1-i}^{i-1}=\Pi_{l=2}^{i}\left({ }^{J} \epsilon_{j-1}^{1}-{ }^{J} \epsilon_{l}^{1}\right), \quad \sum_{J=1}^{P} N_{J}=n+1
$$

and

$$
a_{j}^{J}=1 / \Pi_{k \neq j}\left({ }^{J} \epsilon_{j-1}^{1}-{ }^{J} \epsilon_{k-1}^{1}\right), \quad k=1, \ldots, N_{J},
$$

the fundamental formula (1.9) assumes the form

$$
\sum_{K=1}^{P} \sum_{l=2}^{N_{K}+1} \frac{S_{l}^{K}}{\left(u-e_{K}\right)^{N_{K}+2-l}}=\frac{\Pi_{i=1}^{n}\left(u-u_{i}\right)}{\Pi_{K=1}^{P}\left(u-e_{K}\right)^{N_{K}}},
$$


where $S_{j+1}^{J}=\sum_{i=1}^{j} x_{i}^{J} x_{j+1-i}^{J}$. The expressions for $S_{i}\left[u_{1}, \ldots, u_{n}\right]$ can be determined from this result. Indeed (3.8) can be rewritten

$$
\Pi_{i=1}^{n}\left(u-u_{i}\right)=\sum_{K=1}^{P} \sum_{l=2}^{N_{K}+1} S_{l}^{K}\left(u-e_{k}\right)^{l-2} \Pi_{L \neq K}\left(u-e_{L}\right)^{N_{L}} .
$$

We then deduce that

$$
S_{m}\left[u_{1}, \ldots, u_{n}\right]=\sum_{K=1}^{P} \sum_{l=2}^{N_{K}+1} S_{l}^{K}(-1)^{A_{K}+l+2-n} S_{A_{K}+l-2+m-n}\left[e_{1}\left(N_{1}\right), \ldots, e_{K}(l-2), \ldots, e_{P}\left(N_{P}\right)\right],
$$

where $A_{K}=\sum_{L \neq K} N_{L}$. The coordinates determined in this way are said to be generic coordinates of type $\left\{N_{1}, \ldots, N_{P}\right\}$. Here,

$$
d s^{2}=\sum_{k=1}^{n} \frac{\Pi_{m \neq i}\left(u_{m}-u_{i}\right)}{\prod_{l=1}^{P}\left(u_{k}-e_{l}\right)^{N_{l}}} d u_{k}^{2}
$$

By cross multiplying in the expression (3.8) we see immediately that

$$
\sum_{J=1}^{P} \sum_{i=1}^{N_{J}} x_{i}^{J} x_{N_{J}+1-i}^{J}=1
$$

and the coordinates $x_{j}^{J}$ are given by

$$
S_{j+1}^{J}=\frac{1}{(j-1) !}\left(\frac{\partial}{\partial e_{J}}\right)^{j-1} \frac{\Pi_{i=1}^{n}\left(u_{i}-e_{J}\right)}{\Pi_{L \neq J}\left(e_{L}-e_{J}\right)},
$$

where $S_{j+1}^{J}=\sum_{i=1}^{j} x_{i}^{J} x_{j+1-i}^{J}$.

The case of generic coordinates in $E_{n}$ can be treated similarly. The only restriction now is that $\sum_{J=1}^{p} N_{J}=n+1$ and

$$
\sum_{i=1}^{n} x_{i}^{2} \rightarrow \sum_{J=1}^{p} \sum_{i=1}^{N_{J}} x_{i}^{J} x_{N_{J}+1-i}^{J}
$$

under the transformation given above.

The rules for constructing all other coordinate systems on the complex $n$ sphere that correspond to orthogonal coordinates are just as in Ref. 18 to which we refer. In the Appendix we give some of the potentials for the simplest cases. For the case of hyperbolic $n$ space there are additional coordinate systems which can be regarded as generic. These correspond to the signatures $\{21 \ldots 1\}$ and $\{31 \ldots 1\}$, as well as the generic case $\{1 \ldots 1\}$ in which $e_{1}=e_{2}^{*}$. The first few potentials which correspond to these cases are given in the Appendix.

For the case of complex Euclidean space similar general coordinate systems correspond to a metric of type (3.6) with $\sum_{j=1}^{P} N_{j} \leqslant n$. The various possible forms of this metric are determined by coordinates via limiting processes similar to those for the $n$ sphere. Making the same change of designation as before viz. $x_{j}^{J}$ and $e_{j}^{J}, j=1, \ldots, N_{J}, J=1, \ldots, P$, and $\sum_{j=1}^{P} N_{j}=n$, we can calculate the properties of the various coordinates in $n$ dimensions by suitable limiting procedures from the generic case in which all $e_{i}, i=1, \ldots, n$, are distinct. The only difference in this case is that the condition $e_{j}^{J} \rightarrow e_{J}+{ }^{J} \epsilon_{j-1}^{1}$ could include the possibility that, say, $e_{1}=\infty$. There are two possible signatures that describe coordinate systems of this type: $\left[N_{1}, \ldots, N_{J}\right]$, and $\left[\begin{array}{c}\infty \\ N_{1}\end{array}, \ldots, N_{J}\right]$. All the calculations involving $\left[N_{1}, \ldots, N_{J}\right]$ look exactly as with the corresponding coordinates on the $n$ 
sphere, i.e., the limiting processes are given by (3.7), the fundamental formula by (3.8), and the symmetric functions by (3.10). The only restriction is that $\sum_{j=1}^{P} N_{j}=n$. For the second type of generic coordinates, which correspond to $\left[\begin{array}{c}\infty \\ N_{1}\end{array}, \ldots, N_{J}\right]$, the coordinates are given by

$$
\begin{array}{cc}
S_{j+1}^{1}=\left.(1 /(j-1) !)(\partial / \partial \epsilon)^{j-1}\left[\left(\Pi_{l=1}^{n}\left(\epsilon u_{l}-1\right)\right) /\left(\epsilon e_{J}-\right)^{N_{J}}\right]\right|_{\epsilon=0}, & j=1, \ldots, N_{1} \\
S_{j+1}^{J}=[1 /(j-1) !]\left(\partial / \partial e_{J}\right)^{(j-1)}\left[\Pi_{i=1}^{n}\left(u_{i}-e_{J}\right) / \Pi_{L \neq J}\left(e_{L}-e_{J}\right)\right], & j=1, \ldots, N_{J},
\end{array}
$$

where $S_{j+1}^{J}$ is defined as above with the extra condition that $x_{1}^{1}=1$. The fundamental form defining these coordinates is

$$
\begin{aligned}
& \left.\left(1 /\left(N_{1}-1\right) !\right)(\partial / \partial \epsilon)^{N_{1}-1}\left[\left(\Pi_{l=1}^{n}\left(\epsilon u_{l}-1\right)\right) /\left((\epsilon u-1)\left(\epsilon e_{J}-1\right)^{N_{J}}\right)\right]\right|_{\epsilon=0}+\sum_{J=2}^{P} \sum_{j=1}^{N_{J}} S_{j+1}^{J} / \\
& \left(u-e_{J}\right)^{N_{J}+1-j}=\Pi_{k=1}^{n}\left(u-u_{k}\right) / \Pi_{J=2}^{P}\left(u-e_{J}\right) .
\end{aligned}
$$

The symmetric functions $S_{l}\left[u_{1}, \ldots, u_{n}\right]$ can be read off from this formula.

\section{FLAT SPACE EMBEDDINGS}

It is possible to extend these ideas to rational Stäckel metrics not necessarily corresponding to spaces of constant curvature. As an example consider the Riemannian space whose metric is defined by

$$
d s^{2}=\left(u_{1}-u_{2}\right)\left[\frac{d u_{1}^{2}}{\prod_{j=1}^{5}\left(u_{1}-e_{j}\right)}-\frac{d u_{2}^{2}}{\prod_{j=1}^{5}\left(u_{2}-e_{j}\right)}\right] .
$$

This space is such that the corresponding Hamilton-Jacobi separation of variables method of solution works. It is indeed a special form of the Stäckel separable form we have given in the introduction. As an analogue of what we have been doing so far, we obtain these coordinates from an embedding in a higher-dimensional flat space. Indeed we can obtain suitable coordinates by considering

$$
x_{i}^{2}=\frac{\left(u_{1}-e_{i}\right)\left(u_{2}-e_{i}\right)}{\Pi_{j \neq i}\left(e_{i}-e_{j}\right)}, \quad i=1, \ldots, 5,
$$

subject to the restrictions

$$
\sum_{i=1}^{5} x_{i}^{2}=0, \quad \sum_{i-1}^{5} e_{i} x_{i}^{2}=0, \quad \sum_{i-1}^{5} e_{i}^{2} x_{i}^{2}=1
$$

We now ask the question: what form does a potential have to take in order that it be a Stäckel multiplier which is expressible as a rational function of the $x_{i}$ variables? If the Stäckel multiplier has the form (1.4), and if it is a polynomial in the $x_{i}$ s, then with $u_{2}=e_{1}$, say, $v(u)$ can be written in terms of the functions

$$
\begin{gathered}
R_{2345}=\sqrt{\Pi_{i=2}^{5}\left(u-e_{i}\right)}, \quad R_{i j k}=\sqrt{\left(u-e_{j}\right)\left(u-e_{k}\right)\left(u-e_{i}\right)}, \quad i, j, k \neq, \\
R_{j k}=\sqrt{\left(u-e_{j}\right)\left(u-e_{k}\right)}, \quad j, k \neq, \quad R_{i}=\sqrt{u-e_{i}}
\end{gathered}
$$

as follows: 


$$
v(u)=B_{2345}(u) R_{2345}+\sum_{i, j, k \neq} B_{i j k}(u) R_{i j k}+\sum_{i, j \neq} B_{i j}(u) R_{i j}+\sum_{i} B_{i}(u) R_{i}+B(u),
$$

where $i, j, k$ can have the values $2,3,4,5$ and all the $B$ functions are rational functions of $u$. If we do this for all possible choices of $u_{2}=e_{3}, e_{4}, e_{5}$, then this can only be consistent if $v(u)$ is a polynomial function of $u$. From the relations

$$
\begin{gathered}
u_{1}+u_{2}=E_{1}\left[e_{1}, e_{2}, e_{3}, e_{4}, e_{5}\right]\left(\sum_{i=1}^{5} e_{i}^{2} x_{i}^{2}\right)-\left(\sum_{i=1}^{5} e_{i}^{3} x_{i}^{2}\right), \\
u_{1} u_{2}=\left(\sum_{i=1}^{5} e_{i}^{4} x_{i}^{2}\right)+E_{1}\left[e_{1}, e_{2}, e_{3}, e_{4}, e_{5}\right]\left(\sum_{i=1}^{5} e_{i}^{3} x_{i}^{2}\right)-E_{2}\left[e_{1}, e_{2}, e_{3}, e_{4}, e_{5}\right]\left(\sum_{i=1}^{5} e_{i}^{2} x_{i}^{2}\right)
\end{gathered}
$$

we can deduce the form of the corresponding potentials in terms of the coordinates $x_{i}$. A similar argument can also be used to show that if the potential is a rational function of the $x_{i}$, then the corresponding function $v(u)$ must also be rational.

These results extend easily to the case of general coordinate systems of this type. Indeed let us consider a Riemannian space with infinitesimal distance

$$
d s^{2}=\sum_{k=1}^{n}\left[\Pi_{m \neq i}\left(u_{m}-u_{i}\right)\right] /\left[\Pi_{l=1}^{p}\left(u_{k}-e_{l}\right)\right] d u_{k}^{2},
$$

where $p>n+1$. A suitable choice of Cartesian coordinates is

$$
x_{i}^{2}=(1 / 4) \Pi_{j=1}^{n}\left(u_{j}-e_{i}\right) / \Pi_{k \neq i}\left(e_{k}-e_{i}\right), \quad i, k=1, \ldots, p .
$$

The subspace spanned by these coordinates is given by the simultaneous equations

$$
\sum_{i=1}^{p} e_{i}^{q} x_{i}^{2}=\delta_{q, p-n-1}, \quad q=1, \ldots, p-n-1 .
$$

The symmetric functions $S_{r}\left[u_{1}, \ldots, u_{n}\right]$ are given by the formulas

$$
S_{r}\left[u_{1}, \ldots, u_{n}\right]=(-1)^{p-n} \sum_{s=0}^{r}(-1)^{s} E_{r-s}\left[e_{1}, \ldots, e_{p}\right] \sum_{k=1}^{p} e_{k}^{p-n-1+s} x_{i}^{2} .
$$

The surfaces thus defined are the intersection of $p-n-1$ quadrics. The result expounded for the particular example given above holds in general for these spaces: If the function $v(u)$ in the corresponding Stäckel multiplier is a polynomial/rational in $u$, the Stäckel multiplier is polynomial/rational in the coordinates $x_{i}$.

As a corollary let us consider a Riemannian space with infinitesimal distance

$$
d s^{2}=\sum_{k=1}^{n}\left[\Pi_{m \neq k}\left(u_{m}-u_{k}\right)\right]\left[\Pi_{j=1}^{q}\left(u_{k}-E_{j}\right)\right] /\left[\Pi_{l=1}^{p}\left(u_{k}-e_{l}\right)\right] d u_{k}^{2} .
$$

This system can be obtained from one of the previous type with infinitesimal distance

$$
d s^{2}=\sum_{k=1}^{n+q}\left[\Pi_{m \neq k}\left(u_{m}-u_{k}\right)\right] /\left[\Pi_{l=1}^{p}\left(u_{k}-e_{l}\right)\right] d u_{k}^{2},
$$


subject to the restrictions $u_{n+1}=E_{1}, \ldots, u_{n+q}=E_{q}$. These can be interpreted as the extra constraints

$$
\sum_{i=1}^{n+p} x_{i}^{2} /\left(E_{j}-e_{i}\right)=0, \quad j=1, \ldots, q
$$

Just as before it is possible to make some of the $e_{i}$ equal. The expressions for the infinitesimal distance, coordinates, and the generating function are essentially the same as given previously for systems of type $\left[N_{1}, \ldots, N_{P}\right]$. The constraints now have the form

$$
\sum_{J=1}^{P} \sum_{k=1}^{N_{J}} S_{N_{J}-1+k}\left[\left(\frac{\partial}{\partial e_{J}}\right)^{N_{J}-k} e_{J}^{q}\right]=\delta_{q, p-n-1}
$$

for $q=1, \ldots, p-n-1$.

The results of this section can be summarized as follows. For Stäckel metrics of type

$$
d s^{2}=\sum_{k=1}^{n}\left[\Pi_{m \neq i}\left(u_{m}-u_{i}\right)\right] /\left[\Pi_{l=1}^{P}\left(u_{k}-e_{l}\right)^{N_{l}}\right] d u_{k}^{2},
$$

an embedding into flat space can be distinguished in two ways.

(1) If $\sum_{l=1}^{P} N_{l}=N \geqslant n$, then the coordinates are given by

$$
S_{j+1}^{J}=[1 /(j-1) !]\left(\frac{\partial}{\partial e_{J}}\right)^{(j-1)}\left[\Pi_{i=1}^{n}\left(u_{i}-e_{J}\right) / \Pi_{L \neq J}\left(e_{L}-e_{J}\right)\right]
$$

where $j=1, \ldots, N_{J}, J=1, \ldots, P$. The fundamental form defining these coordinates is

$$
\sum_{J=1}^{P} \sum_{j=1}^{N_{J}} S_{j+1}^{J} /\left(u-e_{J}\right)^{N_{J}+1-j}=\Pi_{k=1}^{n}\left(u-u_{k}\right) / \Pi_{J=2}^{P}\left(u-e_{J}\right) .
$$

The coordinates $x_{i}, i=1, \ldots, N$, are subject to the constraints

$$
\sum_{J=1}^{P} \sum_{k=1}^{N_{J}} S_{N_{J}-1+k}\left[\left(\frac{\partial}{\partial e_{J}}\right)^{N_{J}-k} e_{J}^{q}\right]=\delta_{q, p-n-1}
$$

(2) If $\Sigma_{l=2}^{P} N_{l}=N \geqslant n$, then the coordinates are given by

$$
\begin{aligned}
& S_{j+1}^{1}=\left.(1 /(j-1) !)\left(\frac{\partial}{\partial \epsilon}\right)^{j-1}\left[\left(\Pi_{l=1}^{n}\left(\epsilon u_{l}-1\right)\right) /\left(\epsilon e_{J}-1\right)^{N_{J}}\right]\right|_{\epsilon=0}, \quad j=1, \ldots, N_{1}, \\
& S_{j+1}^{J}=[1 /(j-1) !]\left(\frac{\partial}{\partial e_{J}}\right)^{(j-1)}\left[\Pi_{i=1}^{n}\left(u_{i}-e_{J}\right) / \Pi_{L \neq J}\left(e_{L}-e_{J}\right)\right], \quad j=1, \ldots, N_{J},
\end{aligned}
$$

where $N_{1}=n-N$ and $x_{1}^{1}=1$. The fundamental form defining these coordinates is

$$
\begin{gathered}
\left.\left(1\left(N_{1}-1\right) !\right)\left(\frac{\partial}{\partial \epsilon}\right)^{N_{1}-1}\left[\left(\Pi_{l=1}^{n}\left(\epsilon u_{l}-1\right)\right) /\left((\epsilon u-1)\left(\epsilon e_{J}-1\right)^{N_{J}}\right)\right]\right|_{\epsilon=0} \\
+\sum_{J=2}^{P} \sum_{j=1}^{N_{J}} S_{j+1}^{J} /\left(u-e_{J}\right)^{N_{J}+1-j}=\Pi_{k=1}^{n}\left(u-u_{k}\right) / \Pi_{J=2}^{P}\left(u-e_{J}\right) .
\end{gathered}
$$


In either case the fundamental formula determines the symmetric functions $S_{l}\left[u_{1}, \ldots, u_{n}\right]$. Hence the possible Stäckel multipliers that give rise to rational potentials in terms of the Cartesian coordinates can be determined.

Metrics of the form

$$
d s^{2}=\sum_{k=1}^{m}\left[\Pi_{m \neq k}\left(u_{m}-u_{k}\right)\right]\left[\Pi_{j=1}^{q}\left(u_{k}-E_{j}\right)^{M_{j}}\right] /\left[\Pi_{l=1}^{p}\left(u_{k}-e_{l}\right)^{N_{l}}\right] d u_{k}^{2}
$$

can be obtained from (4.11) via the requirement that $u_{1}=E_{1}, \ldots, u_{M_{1}}=E_{1}, u_{M_{1}+1}$ $=E_{2}, \ldots, u_{M_{1}+M_{2}}=E_{2}, \ldots, u_{M_{1}+\ldots+M_{q}}=E_{q}$. Making suitable redefinitions of the remaining variables we recover the metric given.

In all three cases we can adapt earlier proofs to establish that separable potentials which are rational in the corresponding Cartesian coordinates are generated by the functions $v(u)=(u$ $-a)^{p}, p=0, \pm 1, \pm 2, \ldots$. What has been achieved here is a generalization of the results of Wojciechowski, ${ }^{19,20}$ who obtained families of separable potentials which corresponded to polynomial potentials on the sphere. Indeed these results provide a comprehensive generalization since we have necessary and sufficient conditions that separable potentials can be obtained which are rational functions of "Cartesian coordinates." These also include the rational family of potentials obtained by Wojciechowski.

\section{FURTHER GENERALIZATIONS}

It is possible to generalize further the results presented thus far. Indeed if we consider Stäckel metrics of the form

$$
d s^{2}=\left(u_{1}-u_{2}\right)\left[\frac{d u_{1}^{2}}{\left(u_{1}-e_{1}\right)\left(u_{1}-e_{2}\right)\left(u_{1}-e_{3}\right)}-\frac{d u_{2}^{2}}{\left(u_{2}-a_{1}\right)\left(u_{2}-a_{2}\right)\left(u_{2}-a_{3}\right)}\right],
$$

the corresponding Hamilton-Jacobi and Schrödinger equations afford a separation of variables and the general form of the potential compatible with separation is

$$
V\left(u_{1}, u_{2}\right)=\frac{v_{1}\left(u_{1}\right)-v_{2}\left(u_{2}\right)}{u_{1}-u_{2}} .
$$

The question we now ask is how much the analogy between what we have already done goes through for metrics like this one? We show that an analogue of the embedding into a suitable flat space can be achieved. For the example given above, a suitable choice of Euclidean coordinates is

$$
\begin{gathered}
z_{1 l_{1}, 2 k_{1}}^{2}=A_{1 l_{1}, 2 k_{1}} u_{1 l_{1}, 2 k_{1}}^{2}=A_{1 l_{1} 2 k_{1}}\left(u_{1}-e_{l_{1}}\right)\left(u_{2}-a_{k_{1}}\right)=A_{1 l_{1} 2 k_{1}} v_{1 l_{1}}^{2} w_{2 k_{1}}^{2}, \\
z_{1 l_{1}}^{2}=A_{1 l_{1}}\left(u_{1}-e_{l_{1}}\right)=A_{1 l_{1}} v_{1 l_{1}}^{2}, \\
z_{2 k_{1}}^{2}=A_{2 k_{1}}\left(u_{2}-a_{k_{1}}\right)=A_{2 k_{1}} w_{1 k_{1}}^{2},
\end{gathered}
$$

where $l_{1}, k_{1}=1,2,3$, and a suitable choice of constants is 


$$
\begin{gathered}
A_{1 l_{1} 2 k_{2}}=\frac{4}{3}\left[\frac{e_{l_{2}}-e_{l_{3}}}{\left(e_{l_{1}}-e_{l_{2}}\right)\left(e_{l_{1}}-e_{l_{3}}\right)}+\frac{a_{k_{2}}-a_{k_{3}}}{\left(a_{k_{1}}-a_{k_{2}}\right)\left(a_{k_{1}}-a_{k_{3}}\right)}\right], \\
A_{1 l_{1}}=\frac{4 e_{l_{1}}-\frac{4}{3}\left(a_{1}+a_{2}+a_{3}\right)}{\left(e_{l_{1}}-e_{l_{2}}\right)\left(e_{l_{1}}-e_{l_{3}}\right)}, \quad A_{2 k_{2}}=\frac{4 a_{k_{1}}-\frac{4}{3}\left(e_{1}+e_{2}+e_{3}\right)}{\left(a_{k_{1}}-a_{k_{2}}\right)\left(a_{k_{1}}-a_{k_{3}}\right)},
\end{gathered}
$$

where $l_{1}, l_{2}, l_{3}$ and $k_{1}, k_{2}, k_{3}$ are even permutations of $1,2,3$.

There are relations between the various Cartesian coordinates thus produced. Indeed

$$
\begin{gathered}
v_{1 l_{1}}^{2}-v_{1 l_{2}}^{2}=e_{l_{2}}-e_{l_{1}}, \quad w_{1 k_{1}}^{2}-w_{1 k_{2}}^{2}=a_{k_{2}}-a_{k_{1}}, \\
\left(a_{k_{4}}-a_{k_{3}}\right)\left(u_{1 l_{1} 2 k_{1}}^{2}-u_{1 l_{1} 2 k_{2}}^{2}\right)+\left(a_{k_{1}}-a_{k_{2}}\right)\left(u_{1 l_{2} 2 k_{3}}^{2}-u_{1 l_{2} 2 k_{4}}^{2}\right)+\left(a_{k_{1}}-a_{k_{2}}\right)\left(a_{k_{4}}-a_{k_{3}}\right)\left(e_{l_{1}}-e_{l_{2}}\right)=0,
\end{gathered}
$$

for $k_{1} \neq k_{2}, k_{3} \neq k_{4}$, and $l_{1} \neq l_{2}$. Clearly, there are similar relations with the $e$ and $a$ interchanged.

For the example metric we are looking at, there are only six independent conditions of this quadratic type. There is also a degree of ambiguity in the choice of the constants $A_{1 l_{1} 2 k_{1}}, A_{1 l_{1}}$, and $A_{2 k_{1}}$. This is the result of the existence of the null forms

$$
\begin{aligned}
& d \omega_{1}^{2}=d u_{11,21}^{2}-d u_{11,23}^{2}-d u_{13,21}^{2}+d u_{13,23}^{2}+\left(a_{1}-a_{3}\right)\left(d u_{11}^{2}-d u_{31}^{2}\right)+\left(e_{1}-e_{3}\right)\left(d u_{21}^{2}-d u_{23}^{2}\right)=0, \\
& d \omega_{2}^{2}=d u_{11,22}^{2}-d u_{11,23}^{2}-d u_{13,22}^{2}+d u_{13,23}^{2}+\left(a_{2}-a_{3}\right)\left(d u_{11}^{2}-d u_{13}^{2}\right)+\left(e_{1}-e_{3}\right)\left(d u_{22}^{2}-d u_{23}^{2}\right)=0, \\
& d \omega_{3}^{2}=d u_{12,21}^{2}-d u_{12,23}^{2}-d u_{13,21}^{2}+d u_{13,23}^{2}+\left(a_{1}-a_{3}\right)\left(d u_{12}^{2}-d u_{13}^{2}\right)+\left(e_{2}-e_{3}\right)\left(d u_{21}^{2}-d u_{23}^{2}\right)=0, \\
& d \omega_{4}^{2}=d u_{12,22}^{2}-d u_{12,23}^{2}-d u_{13,22}^{2}+d u_{13,23}^{2}+\left(a_{2}-a_{3}\right)\left(d u_{11}^{2}-d u_{13}^{2}\right)+\left(e_{2}-e_{3}\right)\left(d u_{22}^{2}-d u_{23}^{2}\right)=0 .
\end{aligned}
$$

Can this example be generalized? The answer is yes. Consider

$$
v_{i l}^{2}=\left(u_{i}-A_{i l}\right), \quad i=1, \ldots, N ; \quad l=1, \ldots, m_{i},
$$

where we also take $m_{i}>N$ for all $i$. We define the symbols

$$
U_{i_{1} l_{i_{1}}, i_{2} l_{i_{2}}, \ldots, i_{k} l_{i_{k}}}=v_{i_{1} l_{i_{1}}}^{2} v_{i_{2} l_{i_{2}}}^{2} \cdots v_{i_{k} l_{i_{k}}}^{2},
$$

where $i_{m} \neq i_{n}$ for $m \neq n$ and $k=1, \ldots, N$. We wish to find Cartesian coordinates such that

$$
z_{i_{1} l_{i_{1}}, i_{2} l_{i_{2}}, \ldots, i_{k} l_{i_{k}}}^{2}=A_{i_{1} l_{i_{1}}, i_{2} l_{i_{2}}, \ldots, i_{k} l_{i_{k}}} U_{i_{1} l_{i_{1}}, i_{2} l_{i_{2}}, \ldots, i_{k} l_{i_{k}}}
$$

and

$$
d s^{2}=\sum_{i_{j}, l_{i_{j}}} d z_{i_{1} l_{i_{1}}, i_{2} l_{i_{2}}, \ldots, i_{k} l_{i_{k}}}^{2}=\sum_{i=1}^{N} \frac{\Pi_{j \neq i}\left(u_{i}-u_{j}\right)}{\prod_{l_{i}=1}^{n_{i}}\left(u_{i}-A_{i l_{1}}\right)} d u_{i}^{2} .
$$

This is a metric in Stäckel form which separates both Hamilton-Jacobi and Schrödinger equations. It is convenient to write $S_{j l_{j}}=1 / \Pi_{m_{j} \neq l_{j}}\left(A_{j l_{j}}-A_{j m_{j}}\right)$ and also $A_{j}=\Sigma_{l_{j}} A_{j l_{j}}$. For these quantities the following identities hold: 


$$
\sum_{l_{j}} S_{j l_{j}} A_{j l_{j}}^{k}=0, \quad 0 \leqslant k \leqslant n_{j}
$$

In order to consider Cartesian coordinates of the form (5.8) so as to produce the metric, we need to determine the the constants $A_{i_{1} l_{i_{1}}, i_{2} l_{i_{2}}, \ldots, i_{k} l_{i_{k}}}$. These will be determined in such a way that for fixed $k$ the corresponding Cartesian coordinates $z_{i_{1} l_{1}, i_{2} l_{i_{2}}, \ldots, i_{k} l_{i_{k}}}$ are orthogonal. If $k=n$, then orthogonality is assured if we have

$$
A_{i_{1} l_{i_{1}}, i_{2} l_{i_{2}}, \ldots, i_{N} l_{i_{N}}}=\sum_{k=1}^{N}(N, k \Lambda) S_{k l_{k}} .
$$

To see this we consider, for instance, the term in $d u_{1} d u_{2}$. The corresponding contribution is $(1 / 4)\left(\sum_{k=1}^{N}\left({ }_{N, k} \Lambda\right) S_{k l_{k}}\right) \Pi_{m=3}^{N}\left(u_{m}-A_{m l_{m}}\right)$. Clearly, from the identities (5.10), this type of term summed on the $l_{j}$ gives zero. In general, the correct formula is

$$
A_{i_{1} l_{i_{1}}, i_{2} l_{i_{2}}, \ldots, i_{k} l_{i}}=\sum_{s=1}^{k} \sum_{q=0 p, s}^{N-k} \Lambda_{i_{k+1}, \ldots, i_{N}}^{s_{1}, \ldots, s_{q}} A_{s_{1}} \cdots A_{s_{q}}\left(A_{i_{j} l_{i_{j}}}\right)^{n-k-q} S_{i_{s} l_{i_{s}}}
$$

where ${ }_{p, s} \Lambda_{i_{k+1}, \ldots, i_{N}}^{s_{1}, \ldots, s_{q}}$ is symmetric in the indices $s_{1}, \ldots, s_{q}$ and $i_{k+1}, \ldots, i_{N}$. Summation on the indices $s_{1}, \ldots, s_{q}, s_{1} \neq s_{2} \neq \cdots \neq s_{q}$ and $\left\{s_{1}, \ldots, s_{q}\right\} \subset\left\{i_{k+1}, \ldots, i_{N}\right\}$ is implied. It remains to find the coefficients ${ }_{p, s} \Lambda_{i_{k+1}, \ldots, i_{N}}^{s_{1}, \ldots, s_{q}}$. Let us consider the coefficient of $d u_{1}^{2}$. We wish to construct the coefficient to be $\Pi_{j \neq 1}\left(u_{1}-u_{j}\right) / \Pi_{l_{1}=1}^{n_{1}}\left(u_{1}-A_{1 l_{1}}\right)$. In particular we consider the term $(-1)^{N-1} u_{2} \cdots u_{N} / \Pi_{l_{1}}\left(u_{1}-A_{1 l_{1}}\right)$. Typical contributing factors to the numerator are $\left(\sum_{k=1 N, k}^{N} \Lambda S_{k l_{k}}\right)\left(\Pi_{m=2}^{N}\left(u_{m}-A_{m l_{m}}\right)\right)$. The coefficient of $(-1)^{N-1} u_{2} \cdots u_{N} /\left[\Pi_{l_{1}}\left(u_{1}-A_{1 l_{1}}\right)\right]$ is $\Sigma_{l_{1}, l_{2}, \ldots, l_{N} N, k} \Lambda S_{1 l_{1}} /\left(u_{1}-A_{1 l_{1}}\right)=n_{2} \cdots n_{N} /\left[\Pi_{l_{1}}\left(u_{1}-A_{1 l_{1}}\right)\right]$ from which it follows that ${ }_{N, 1} \Lambda$ $=(-1)^{N-1} /\left(n_{2} \cdots n_{N}\right)$. Proceeding to the calculation of the coefficient of $u_{3} \cdots u_{N} / \Pi_{l_{1}}\left(u_{1}\right.$ $-A_{1 l_{1}}$ ) we find the contribution of terms of the form (5.12) for $k=N$ is $-A_{2} n_{3}, \ldots, n_{N}(N, 1)$ ). The other contributing term comes from

$$
A_{1 l_{1} 3 l_{3}, \ldots, N l_{N}} U_{1 l_{1} 3 l_{3}, \ldots, N l_{N}}=\sum_{k \neq 2}\left[\left({ }_{N-1, k} \Lambda\right)\left(A_{k l k}\right)+\left({ }_{N-1, k} \Lambda_{2}^{2}\right) A_{2}\right] S_{k l_{k}}
$$

and is ${ }_{N-1,1} \Lambda_{2}^{2}$. Therefore we have ${ }_{N-1,1} \Lambda_{2}^{2}={ }_{N, 1} \Lambda$. Proceeding in this way we can establish that

$$
{ }_{p, i_{1}} \Lambda_{i_{p+1}, \ldots, i_{N}}^{i_{p+1}, \ldots, i_{N}}={ }_{N, i_{1}} \Lambda
$$

Repeating these arguments for the other coefficients we obtain the general formula

$$
\begin{gathered}
N_{N-p, i_{p}} \Lambda_{i_{p+1}, \ldots, i_{N}}=(-1)^{N-p} / \Pi_{k=1}^{p-1} n_{i_{k}}, \\
N-p-q, i_{p} \Lambda_{i_{p+1}, \ldots, i_{N}, r_{1}, \ldots, r_{q}}^{r_{1}, \ldots, r_{q}}={ }_{N-p, i_{p}} \Lambda_{i_{p+1}, \ldots, i_{N} .} .
\end{gathered}
$$

This completes the embedding of the coordinates $v_{i l_{i}}$ into a flat space of dimension $\Pi_{k=1}^{N}\left(n_{k}+1\right)$. The various relations among the coordinates are consequences of the relations $v_{i l_{i}}^{2}-v_{j l_{j}}^{2}=A_{j l_{j}}-A_{i l_{i}}$. The choice of coordinates given above is unique modulo quadratic forms which are null and diagonal. The set of all such forms is generated by coefficients given by 


$$
A_{i_{1} l_{i_{1}}, \ldots, i_{k} l_{i_{k}}}=(-1)^{c_{k}}, \quad A_{i_{s} l_{i_{s}}}=\Pi_{j \neq s}\left(a_{i_{j}}-b_{i_{j}}\right) \epsilon_{l_{i_{s}}},
$$

where $l_{i_{s}}=a_{i_{s}}$ or $b_{i_{s}}$ and $\epsilon_{i_{s}}=1$ if $l_{i_{s}}=a_{i_{s}}$ and $\epsilon_{i_{s}}=-1$ if $l_{i_{s}}=b_{i_{s}}$. Further, $c_{k}=\sum_{j=1}^{k} \epsilon_{l_{i_{j}}}$.

Separable potentials that are polynomials are constructed in the same way as previously from the symmetric functions $S_{l}\left[u_{1}, \ldots, u_{n}\right]$. In particular if we define

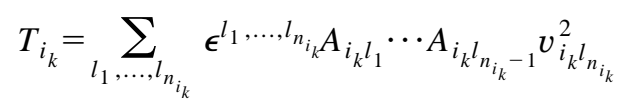

and

$$
P_{i_{k}}=\Pi_{l_{i_{k}}>m_{i_{k}}}\left(A_{i_{k} l_{i_{k}}}-A_{i_{k} m_{i_{k}}}\right),
$$

then

$$
\Pi_{j=1}^{k} u_{i_{j}}=\Pi_{j=1}^{k} \frac{T_{i_{j}}}{P_{i_{j}}} .
$$

In the above formula $\epsilon^{l_{1} \cdots l_{q}}$ is the totally skew-symmetric tensor. From these formulas, expressions can be constructed for the symmetric functions in terms of quadratic functions of the $x_{i} \mathrm{~s}$. If we look for potentials which are rational functions of the $x_{i}$ s, then the restriction to symmetric functions is no longer necessary. In fact all that is necessary is that the corresponding Stäckel multiplier be a rational function of the variables $u_{i}$.

It is also possible to consider metrics for which some of the constants $A_{i l_{i}}$ are equal. As an example, consider the coordinates defined by

$$
\begin{gathered}
\omega_{11,2 l_{2}}^{2}=\frac{\left(u_{1}-e_{1}\right)\left(u_{2}-a_{l_{2}}\right)}{\left(e_{1}-e_{3}\right)}, \\
2 \omega_{11,2 l_{2}} \omega_{12,2 l_{2}}=\frac{2\left(u_{1}-e_{1}\right)\left(u_{2}-a_{l_{2}}\right)}{\left(a_{l_{3}}-a_{l_{2}}\right)\left(a_{l_{3}}-a_{l_{2}}\right)}+\frac{\partial}{\partial e_{1}} \frac{\left(u_{1}-e_{1}\right)\left(u_{2}-a_{l_{2}}\right)}{\left(e_{1}-e_{3}\right)}, \\
\omega_{13,2 l_{2}}^{2}=\left[\frac{-1}{\left(e_{3}-e_{2}\right)^{2}}+\frac{-1}{\left(a_{l_{3}}-a_{l_{2}}\right)\left(a_{l_{3}}-a_{l_{2}}\right)}\right]\left(u_{1}-e_{3}\right)\left(u_{2}-a_{l_{2}}\right), \\
\omega_{11}^{2}=\left[4\left(e_{1}-(1 / 3)\left(a_{1}+a_{2}+a_{3}\right)\right)\left(u_{1}-e_{1}\right) /\left(e_{1}-e_{3}\right)\right], \\
2 \omega_{11} \omega_{12}=\frac{\partial}{\partial e_{1}} \frac{4\left(e_{1}-\frac{1}{3}\left(a_{1}+a_{2}+a_{3}\right)\right)\left(u_{1}-e_{1}\right)}{\left(e_{1}-e_{3}\right)}, \\
\omega_{13}^{2}=\frac{4\left(e_{3}-\frac{1}{3}\left(a_{1}+a_{2}+a_{3}\right)\right)\left(u_{1}-e_{3}\right)}{\left(e_{1}-e_{3}\right)^{2}}, \\
\omega_{2 l_{2}}^{2}=\frac{4\left(a_{l_{2}}-\frac{1}{3}\left(2 e_{1}+e_{3}\right)\right)\left(u_{2}-a_{l_{2}}\right)}{\left(a_{l_{3}}-a_{l_{2}}\right)\left(a_{l_{3}}-a_{l_{2}}\right)} .
\end{gathered}
$$

The corresponding infinitesimal distance is 


$$
\begin{aligned}
d s^{2} & =\sum_{l_{2}=1}^{3}\left(2 d \omega_{11,2 l_{2}} d \omega_{12,2 l_{2}}+d \omega_{13,2 l_{2}}^{2}+d \omega_{2 l_{2}}^{2}\right)+2 d \omega_{11} d \omega_{12}+d \omega_{13}^{2} \\
& =\left(u_{1}-u_{2}\right)\left[\frac{d u_{1}^{2}}{\left(u_{1}-e_{1}\right)^{2}\left(u_{1}-e_{3}\right)}-\frac{d u_{2}^{2}}{\left(u_{2}-a_{1}\right)\left(u_{2}-a_{2}\right)\left(u_{2}-a_{3}\right)}\right] .
\end{aligned}
$$

It is also possible to lift the restriction $m_{i}>N$. We illustrate this with the following example. If we choose coordinates $\omega_{i}$ such that

$$
\begin{gathered}
\omega_{1}^{2}=\left(u_{2}-a_{3}\right)^{2}, \quad \omega_{1} \omega_{2}=2\left(u_{2}-a_{3}\right) u_{1}, \\
\omega_{3}^{2}=u_{2}-a_{3}, \quad \omega_{3} \omega_{4}=\left(u_{2}-a_{3}\right)\left(-1+4\left(u_{2}-a_{3}\right)\right), \\
\omega_{5}^{2}=u_{1}-e_{2}, \quad \omega_{5} \omega_{6}=\frac{\left(u_{1}-e_{2}\right)\left(-5 u_{2}+4 e_{2}+a_{3}\right)}{e_{2}-e_{3}}, \\
\omega_{7}^{2}=u_{1}-e_{3}, \quad \omega_{7} \omega_{8}=\frac{\left(u_{1}-e_{3}\right)\left(-5 u_{2}+4 e_{3}+a_{3}\right)}{e_{3}-e_{2}}, \\
\omega_{9}^{2}=-\left(u_{1}-e_{2}\right)\left(u_{2}-a_{3}\right) /\left(e_{3}-e_{2}\right), \quad \omega_{10}^{2}=-\left(u_{1}-e_{3}\right)\left(u_{2}-a_{3}\right) /\left(e_{2}-e_{3}\right), \quad \omega_{11}^{2}=u_{2}^{2},
\end{gathered}
$$

the corresponding infinitesimal distance is

$$
\begin{aligned}
d s^{2} & =d \omega_{1} d \omega_{2}+d \omega_{3} d \omega_{4}+d \omega_{5} d \omega_{6}+d \omega_{7} d \omega_{8}+d \omega_{9}^{2}+d \omega_{10}^{2}+d \omega_{11}^{2} \\
& =2\left(u_{1}-u_{2}\right)\left[\frac{d u_{1}^{2}}{\left(u_{1}-e_{1}\right)\left(u_{1}-e_{3}\right)}-\frac{d u_{2}^{2}}{u_{2}-a_{3}}\right] .
\end{aligned}
$$

\section{ACKNOWLEDGMENT}

W.M. was supported in part by the National Science Foundation under Grant No. DMS 94-00533.

\section{APPENDIX}

Here we adopt the shortened notation $S_{i}=S_{i}\left[u_{1}, \ldots, u_{n}\right]$ and work out a few specific examples of the properties that have been developed above. For the expressions for the Stäckel multipliers we have

$$
\begin{gathered}
S M_{n-1}=1, \quad S M_{n}=S_{1}, \quad S M_{n+1}=S_{1}^{2}-S_{2}, \\
S M_{n+2}=S_{1}^{3}-2 S_{1} S_{2}+S_{3}, \quad S M_{n+3}=S_{1}^{4}-3 S_{1}^{2} S_{2}+2 S_{1} S_{3}+S_{2}^{2}-S_{4} .
\end{gathered}
$$




\section{Elliptical coordinates in Euclidean $\boldsymbol{n}$ space}

In this case the functions $S_{i}$ are given by (1.8). Consequently,

$$
\begin{gathered}
S M_{n}=E_{1}\left[e_{1}, \ldots, e_{n}\right]+\sum_{k=1}^{n} x_{k}^{2}, \\
S M_{n+1}=\left(E_{1}\left[e_{1}, \ldots, e_{n}\right]+\sum_{k=1}^{n} x_{k}^{2}\right)^{2}-\left(E_{2}\left[e_{1}, \ldots, e_{n}\right]+\sum_{k=1}^{n} x_{k}^{2} E_{1}\left[e_{1}, \ldots, e_{k-1}, e_{k+1}, \ldots, e_{n}\right]\right), \\
S M_{n+2}=\left(E_{1}\left[e_{1}, \ldots, e_{n}\right]+\sum_{k=1}^{n} x_{k}^{2}\right)^{3}-2\left(E_{1}\left[e_{1}, \ldots, e_{n}\right]+\sum_{k=1}^{n} x_{k}^{2}\right) \\
\times\left(E_{2}\left[e_{1}, \ldots, e_{n}\right]+\sum_{k=1}^{n} x_{k}^{2} E_{1}\left[e_{1}, \ldots, e_{k-1}, e_{k+1}, \ldots, e_{n}\right]\right) \\
+\left(E_{3}\left[e_{1}, \ldots, e_{n}\right]+\sum_{k=1}^{n} x_{k}^{2} E_{2}\left[e_{1}, \ldots, e_{k-1}, e_{k+1}, \ldots, e_{n}\right]\right)
\end{gathered}
$$

\section{Paraboloidal coordinates in $E_{n}$}

In this case the functions $S_{i}$ are given by (1.13). Consequently,

$$
\begin{gathered}
S M_{n}=-2 x_{1}+E_{1}\left[e_{2}, \ldots, e_{n}\right], \\
S M_{n+1}=\left(-2 x_{1}+E_{1}\left[e_{2}, \ldots, e_{n}\right]\right)^{2}-\left(E_{2}\left[e_{2}, \ldots, e_{n}\right]-2 x_{1} E_{1}\left[e_{2}, \ldots, e_{n}\right]-\sum_{k=2}^{n} x_{k}^{2}\right), \quad(\mathrm{A} 3) \\
S M_{n+2}=\left(-2 x_{1}+E_{1}\left[e_{2}, \ldots, e_{n}\right]\right)^{3}-2\left(-2 x_{1}+E_{1}\left[e_{2}, \ldots, e_{n}\right]\right)\left(E_{2}\left[e_{2}, \ldots, e_{n}\right]-2 x_{1} E_{1}\left[e_{2}, \ldots, e_{n}\right]\right. \\
\left.-\sum_{k=2}^{n} x_{k}^{2}\right)+\left(E_{3}\left[e_{2}, \ldots, e_{n}\right]-2 x_{1} E_{2}\left[e_{2}, \ldots, e_{n}\right]-\sum_{k=2}^{n} E_{1}\left[e_{2}, \ldots, e_{k-1}, e_{k+1}, \ldots, e_{n}\right] x_{k}^{2}\right) .
\end{gathered}
$$

For the $n$ sphere the corresponding Stäckel multipliers are given by

$$
\begin{gathered}
S M_{n}=\sum_{k=1}^{n+1} E_{1}\left[e_{1}, \ldots, e_{k-1}, e_{k+1}, \ldots, e_{n+1}\right] x_{k}^{2}, \\
S M_{n+1}=\left(\sum_{k=1}^{n+1} E_{1}\left[e_{1}, \ldots, e_{k-1}, e_{k+1}, \ldots, e_{n+1}\right] x_{k}^{2}\right)^{2}-\sum_{k=1}^{n+1} E_{2}\left[e_{1}, \ldots, e_{k-1}, e_{k+1}, \ldots, e_{n+1}\right] x_{k}^{2}, \\
S M_{n+2}=\left(\sum_{k=1}^{n+1} E_{1}\left[e_{1}, \ldots, e_{k-1}, e_{k+1}, \ldots, e_{n+1}\right] x_{k}^{2}\right)^{3}-2\left(\sum_{k=1}^{n+1} E_{1}\left[e_{1}, \ldots, e_{k-1}, e_{k+1}, \ldots, e_{n+1}\right] x_{k}^{2}\right) \\
\times\left(\sum_{k=1}^{n+1} E_{2}\left[e_{1}, \ldots, e_{k-1}, e_{k+1}, \ldots, e_{n+1}\right] x_{k}^{2}\right)+\left(\sum_{k=1}^{n+1} E_{3}\left[e_{1}, \ldots, e_{k-1}, e_{k+1}, \ldots, e_{n+1}\right] x_{k}^{2}\right) .
\end{gathered}
$$


The basic building blocks for rational potentials in the generic elliptic coordinate systems are obtained from the Stäckel multipliers of the form (2.2) with $v(u)=u^{-p}$ where $p$ is a positive integer. We list the first few such potentials. In terms of the functions $S_{i}$ we have

$$
\begin{gathered}
S M_{-1}=\frac{1}{S_{n}}, \quad S M_{-2}=\frac{S_{n-1}}{S_{n}^{2}}, \quad S M_{-3}=\frac{\left(S_{n-1}^{2}-S_{n} S_{n-2}\right)}{S_{n}^{3}} \\
S M_{-4}=\frac{\left(S_{n-1}^{3}-2 S_{n} S_{n-1} S_{n-2}+S_{n-3} S_{n}^{2}\right)}{S_{n}^{4}}
\end{gathered}
$$

For generic elliptical coordinates in Euclidean $n$ space these potentials have the form

$$
\begin{aligned}
S M_{-1}= & \left(E_{n}\left[e_{1}, \ldots, e_{n}\right]+\sum_{k=1}^{n} x_{k}^{2} E_{n-1}\left[e_{1}, \ldots, e_{k-1}, e_{k+1}, \ldots, e_{n}\right]\right)^{-1}, \\
S M_{-2}= & \frac{E_{n-1}\left[e_{1}, \ldots, e_{n}\right]+\sum_{k=1}^{n} x_{k}^{2} E_{n-2}\left[e_{1}, \ldots, e_{k-1}, e_{k+1}, \ldots, e_{n}\right]}{\left(E_{n}\left[e_{1}, \ldots, e_{n}\right]+\sum_{k=1}^{n} x_{k}^{2} E_{n-1}\left[e_{1}, \ldots, e_{k-1}, e_{k+1}, \ldots, e_{n}\right]\right)^{2}}, \\
S M_{-3}= & \left(\begin{array}{l}
\left.E_{n-1}\left[e_{1}, \ldots, e_{n}\right]+\sum_{k=1}^{n} x_{k}^{2} E_{n-2}\left[e_{1}, \ldots, e_{k-1}, e_{k+1}, \ldots, e_{n}\right]\right)^{2} \\
\end{array}\right. \\
& -\left(E_{n}\left[e_{1}, \ldots, e_{n}\right]+\sum_{k=1}^{n} x_{k}^{2} E_{n-1}\left[e_{1}, \ldots, e_{k-1}, e_{k+1}, \ldots, e_{n}\right]\right)^{n} \\
& \left.\times\left(E_{n-2}\left[e_{1}, \ldots, e_{n}\right]+\sum_{k=1}^{n} x_{k}^{2} E_{n-3}\left[e_{1}, \ldots, e_{k-1}, e_{k+1}, \ldots, e_{n}\right]\right)\right) \\
& \left(\begin{array}{l}
\left.\left.E_{1}, \ldots, e_{n}\right]+\sum_{k=1}^{2} E_{n-1}\left[e_{1}, \ldots, e_{k-1}, e_{k+1}, \ldots, e_{n}\right]\right) \\
E_{k}
\end{array}\right)
\end{aligned}
$$

For paraboloidal coordinates in Euclidean $n$ space these potentials have the form 


$$
\begin{aligned}
& S M_{-1}=-\left(2 x_{1} E_{n-1}\left[e_{2}, \ldots, e_{n}\right]+\sum_{k=2}^{n} x_{k}^{2} E_{n-2}\left[e_{2}, \ldots, e_{k-1}, e_{k+1}, \ldots, e_{n}\right]\right)^{-1}, \\
S M_{-2}= & \left(E_{n-1}\left[e_{1}, \ldots, e_{n}\right]-2 x_{1} E_{n-2}\left[e_{1}, \ldots, e_{n}\right]-\sum_{k=2}^{n} x_{k}^{2} E_{n-3}\left[e_{2}, \ldots, e_{k-1}, e_{k+1}, \ldots, e_{n}\right]\right) / \\
& \left(-2 x_{1} E_{n-1}\left[e_{2}, \ldots, e_{n}\right]-\sum_{k=2}^{n} x_{k}^{2} E_{n-2}\left[e_{2}, \ldots, e_{k-1}, e_{k+1}, \ldots, e_{n}\right]\right)^{2}, \\
S M_{-3}= & \left(\left(E_{n-1}\left[e_{1}, \ldots, e_{n}\right]-2 x_{1} E_{n-2}\left[e_{1}, \ldots, e_{n}\right]-\sum_{k=2}^{n} x_{k}^{2} E_{n-3}\left[e_{2}, \ldots, e_{k-1}, e_{k+1}, \ldots, e_{n}\right]\right)^{2}\right. \\
& -\left(-2 x_{1} E_{n-1}\left[e_{2}, \ldots, e_{n}\right]-\sum_{k=2}^{n} x_{k}^{2} E_{n-2}\left[e_{2}, \ldots, e_{k-1}, e_{k+1}, \ldots, e_{n}\right]\right)\left(E_{n-2}\left[e_{1}, \ldots, e_{n}\right]\right. \\
& \left.\left.-2 x_{1} E_{n-3}\left[e_{1}, \ldots, e_{n}\right]-\sum_{k=2}^{n} x_{k}^{2} E_{n-4}\left[e_{2}, \ldots, e_{k-1}, e_{k+1}, \ldots, e_{n}\right]\right)\right) / \\
& \left(-2 x_{1} E_{n-1}\left[e_{2}, \ldots, e_{n}\right]-\sum_{k=2}^{n} x_{k}^{2} E_{n-2}\left[e_{2}, \ldots, e_{k-1}, e_{k+1}, \ldots, e_{n}\right]\right)
\end{aligned}
$$

For the corresponding coordinates on the $n$ sphere

$$
\begin{gathered}
S M_{-1}=\left(\sum_{k=1}^{n+1} x_{k}^{2} E_{n}\left[e_{1}, \ldots, e_{k-1}, e_{k+1}, \ldots, e_{n}\right]\right)^{-1}, \\
S M_{-2}=\frac{\sum_{k=1}^{n+1} x_{k}^{2} E_{n-1}\left[e_{1}, \ldots, e_{k-1}, e_{k+1}, \ldots, e_{n}\right]}{\left(\sum_{k=1}^{n+1} x_{k}^{2} E_{n}\left[e_{1}, \ldots, e_{k-1}, e_{k+1}, \ldots, e_{n}\right]\right)^{2}}, \\
S M_{-3}=\left(\left(\sum_{k=1}^{n+1} x_{k}^{2} E_{n-1}\left[e_{1}, \ldots, e_{k-1}, e_{k+1}, \ldots, e_{n}\right]\right)^{2}-\sum_{k=1}^{n+1} x_{k}^{2} E_{n}\left[e_{1}, \ldots, e_{k-1}, e_{k+1}, \ldots, e_{n}\right]\right) \\
\times\left(\sum_{k=1}^{n+1} x_{k}^{2} E_{n-2}\left[e_{1}, \ldots, e_{k-1}, e_{k+1}, \ldots, e_{n}\right]\right) /\left(\sum_{k=1}^{n+1} x_{k}^{2} E_{n}\left[e_{1}, \ldots, e_{k-1}, e_{k+1}, \ldots, e_{n}\right]\right)^{3} .
\end{gathered}
$$

As noted previously there are additional types of generic coordinate systems associated with the $n$-dimensional hyperboloid. We here list the expressions for the symmetric functions $S_{i}\left[e_{1}, \ldots, e_{n}\right]$ in terms of the corresponding coordinates $x_{k}$.

Case 1:

$$
\begin{aligned}
S_{i}\left[u_{1}, \ldots, u_{n}\right]= & -E_{i-1}\left[e_{3}, \ldots, e_{n+1}\right] x_{1}^{2}+E_{i}\left[e_{1}, e_{3}, \ldots, e_{n+1}\right] 2 x_{1} x_{2} \\
& +\sum_{k=3}^{n+1} E_{i}\left[e_{1}(2), e_{3}, \ldots, e_{k-1}, e_{k+1}, \ldots, e_{n+1}\right]
\end{aligned}
$$

for coordinates of type $\{21 \ldots 1\}$. 
Case 2:

$$
\begin{aligned}
S_{i}\left[u_{1}, \ldots, u_{n}\right]= & E_{i-2}\left[e_{4}, \ldots, e_{n+1}\right] x_{1}^{2}-E_{i-1}\left[e_{1}, e_{3}, \ldots, e_{n+1}\right] 2 x_{1} x_{2}+E_{i}\left[e_{1}(2), e_{4}, \ldots, e_{n+1}\right] \\
& \times\left(x_{2}^{2}+2 x_{1} x_{3}\right)+\sum_{k=4}^{n+1} E_{i}\left[e_{1}(3), e_{4}, \ldots, e_{k-1}, e_{k+1}, \ldots, e_{n+1}\right]
\end{aligned}
$$

for coordinates of type $\{31 \ldots 1\}$. These expressions could of course be substituted into the expressions for the Stäckel multipliers given above. The relation of the coordinates $x_{k}$ to the standard coordinates on the hyperboloid can be deduced from the expressions $2 x_{1} x_{2}+\sum_{k=3}^{n+1} x_{k}^{2}=1$ for the case $\{21 \ldots 1\}$ and $x_{2}^{2}+2 x_{1} x_{3}+\sum_{k=4}^{n+1} x_{k}^{2}=1$ for the $\{31 \ldots 1\}$. Indeed, in case 1 choose coordinates such that $x_{1}=\left(y_{1}-y_{2}\right) / \sqrt{2}, x_{2}=\left(y_{1}+y_{2}\right) / \sqrt{2}, x_{k}=i y_{k}, k=3, \ldots, n+1$, and in case 2 choose coordinates such that $x_{1}=\left(y_{1}-y_{3}\right) / \sqrt{2}, x_{2}=i y_{2}, x_{3}=\left(y_{1}+y_{3}\right) / \sqrt{2}, x_{k}=i y_{k}, k=3, \ldots, n+1$. With these coordinates we have $y_{1}^{2}-\sum_{k=2}^{n+1} y_{k}^{2}=1$. For $n=3$ we give the expressions for the series of Stäckel multipliers.

(1) Coordinates of type $\{111\}$ :

$$
\begin{gathered}
S M_{2}=\left(e_{2}+e_{3}\right) x_{1}^{2}+\left(e_{2}+e_{1}\right) x_{2}^{2}+\left(e_{2}+e_{1}\right) x_{3}^{2}, \\
S M_{3}=\left(\left(e_{2}+e_{3}\right) x_{1}^{2}+\left(e_{2}+e_{1}\right) x_{2}^{2}+\left(e_{2}+e_{1}\right) x_{3}^{2}\right)^{2}-\left(e_{2} e_{3} x_{1}^{2}+e_{2} e_{1} x_{2}^{2}+e_{2} e_{1} x_{3}^{2}\right) \\
S M_{-1}=\left(e_{2} e_{3} x_{1}^{2}+e_{2} e_{1} x_{2}^{2}+e_{2} e_{1} x_{3}^{2}\right)^{-1} \\
S M_{-2}=\left(e_{2}+e_{3}\right) x_{1}^{2}+\left(e_{2}+e_{1}\right) x_{2}^{2}+\frac{\left(e_{2}+e_{1}\right) x_{3}^{2}}{\left(e_{2} e_{3} x_{1}^{2}+e_{2} e_{1} x_{2}^{2}+e_{2} e_{1} x_{3}^{2}\right)^{2}}, \\
S M_{-3}=\left(\left(e_{2}+e_{3}\right) x_{1}^{2}+\left(e_{2}+e_{1}\right) x_{2}^{2}+\left(e_{2}+e_{1}\right) x_{3}^{2}\right)^{2}-\left(e_{2} e_{3} x_{1}^{2}+e_{2} e_{1} x_{2}^{2}+e_{2} e_{1} x_{3}^{2}\right) / \\
\left(e_{2} e_{3} x_{1}^{2}+e_{2} e_{1} x_{2}^{2}+e_{2} e_{1} x_{3}^{2}\right)^{3} .
\end{gathered}
$$

(2) Coordinates of type $\{21\}$ :

$$
\begin{gathered}
S M_{2}=-x_{1}^{2}+2\left(e_{1}+e_{3}\right) x_{1} x_{2}+2 e_{1} x_{3}^{2}, \\
S M_{3}=\left(-x_{1}^{2}+2\left(e_{1}+e_{3}\right) x_{1} x_{2}+2 e_{1} x_{3}^{2}\right)^{2}-\left(2 e_{1} e_{3} x_{1} x_{2}-e_{3} x_{1}^{2}+e_{1}^{2} x_{3}^{2}\right) \\
S M_{-1}=\left(2 e_{1} e_{3} x_{1} x_{2}-e_{3} x_{1}^{2}+e_{1}^{2} x_{3}^{2}\right)^{-1}, \\
S M_{-2}=\frac{-x_{1}^{2}+2\left(e_{1}+e_{3}\right) x_{1} x_{2}+2 e_{1} x_{3}^{2}}{\left(2 e_{1} e_{3} x_{1} x_{2}-e_{3} x_{1}^{2}+e_{1}^{2} x_{3}^{2}\right)^{2}} \\
S M_{-3}=\frac{\left(-x_{1}^{2}+2\left(e_{1}+e_{3}\right) x_{1} x_{2}+2 e_{1} x_{3}^{2}\right)^{2}-\left(2 e_{1} e_{3} x_{1} x_{2}-e_{3} x_{1}^{2}+e_{1}^{2} x_{3}^{2}\right)}{\left(2 e_{1} e_{3} x_{1} x_{2}-e_{3} x_{1}^{2}+e_{1}^{2} x_{3}^{2}\right)^{3}} .
\end{gathered}
$$

(3) Coordinates of type $\{3\}$ : 


$$
\begin{gathered}
S M_{2}=-2 x_{1} x_{2}+2 e_{1}\left(x_{2}^{2}+2 x_{1} x_{3}\right), \\
S M_{3}=\left(-2 x_{1} x_{2}+2 e_{1}\left(x_{2}^{2}+2 x_{1} x_{3}\right)\right)^{2}-\left(e_{1}^{2}\left(x_{2}^{2}+2 x_{1} x_{3}\right)+x_{1}^{2}-2 e_{1} x_{1} x_{2}\right), \\
S M_{-1}=\left(e_{1}^{2}\left(x_{2}^{2}+2 x_{1} x_{3}\right)+x_{1}^{2}-2 e_{1} x_{1} x_{2}\right)^{-1}, \\
S M_{-2}=\frac{-2 x_{1} x_{2}+2 e_{1}\left(x_{2}^{2}+2 x_{1} x_{3}\right)}{\left(e_{1}^{2}\left(x_{2}^{2}+2 x_{1} x_{3}\right)+x_{1}^{2}-2 e_{1} x_{1} x_{2}\right)^{2}}, \\
S M_{-3}=\left(-2 x_{1} x_{2}+2 e_{1}\left(x_{2}^{2}+2 x_{1} x_{3}\right)\right)^{2}-\frac{\left.e_{1}^{2}\left(x_{2}^{2}+2 x_{1} x_{3}\right)+x_{1}^{2}-2 e_{1} x_{1} x_{2}\right)}{\left(e_{1}^{2}\left(x_{2}^{2}+2 x_{1} x_{3}\right)+x_{1}^{2}-2 e_{1} x_{1} x_{2}\right)^{3}} .
\end{gathered}
$$

${ }^{1}$ C. Neumann, "De problemate quodan mechanico, quod ad priman integralium ultraellipticorum classen revocatur," J. Reine Angew. Math 56, 46-63 (1859).

${ }^{2}$ E. Jacobi, Vorlesungen über Dynamik (Supplement band, Berlin, 1884).

${ }^{3}$ J. Moser, "Various aspects of integrable Hamiltonian systems," in Dynamical Systems (Birkhauser, Boston MA, 1980).

${ }^{4}$ A. M. Perelomov, Integrable systems of classical mechanics and Lie algebras. Vol. 1 (Birkhauser, Boston, MA, 1990).

${ }^{5}$ E. G. Kalnins and W. Miller, Jr., "Separation of variables on $n$-dimensional Riemannian manifolds I. The $n$-sphere and Euclidean $n$-space,’’ J. Math. Phys. 27, 1721-1736 (1986).

${ }^{6}$ E. G. Kalnins, "Separation of Variables for Riemannian Spaces of Constant Curvature," in Pitman Monographs and Surveys in Pure and Applied Mathematics, No. 28 (Longman Scientific and Technical, Essex, England, 1986).

${ }^{7}$ C. P. Boyer, E. G. Kalnins, and W. Miller, Jr., “'Stäckel-equivalent integrable Hamiltonian systems,"' SIAM J. Math. Anal 17, 778-797 (1986).

${ }^{8}$ P. Stäckel, "Über die integration der Hamilton-Jacobischen differentialgeichung mittels separation der variabeln," (Habilitationschrift, Halle 1891).

${ }^{9}$ L. P. Eisenhart, "Separable systems of Stäckel," Ann. Math. 35, 284-305 (1934).

${ }^{10}$ E. G. Kalnins and W. Miller, Jr., "Killing tensors and variable separation for Hamilton-Jacobi and Helmholtz equations," SIAM J. Math. Anal. 11, 1011-1026 (1980).

${ }^{11}$ S. Benenti, "Separability structures on Riemannian manifolds," in Proceedings of the Conference on Differential Geometric Methods in Mathematical Physics, Lecture Notes in Mathematics (Springer-Verlag, Berlin, 1980), Vol. 836.

${ }^{12}$ V. N. Shapovalov, "Stäckel spaces," Siberian Math. J. 20, 790-800 (1980).

${ }^{13}$ E. G. Kalnins and W. Miller, Jr., "Killing tensors and nonorthogonal variable separation for the Hamilton-Jacobi equation," SIAM J. Math. Anal. 12, 617-638 (1981).

${ }^{14}$ E. G. Kalnins and W. Miller, "The general theory of $R$-separation for Helmholtz equations," J. Math. Phys. 24, 1047-1053 (1983).

${ }^{15}$ E. G. Kalnins and W. Miller, Jr., "The theory of orthogonal $R$-separation for Helmholtz equations,” Adv. Math. 51, 91-106 (1984).

${ }^{16}$ S. Benenti, “'Orthogonal Separable Dynamical Systems," in Proceedings of the 5th International Conference on Differential Geometry and its Applications, 1992, edited by O. Kowalski and D. Krupka, Mathematical Publications Vol. 1 (Silesian University at Opava, Opava, 1993).

${ }^{17}$ J. Moser, Geometry of quadrics and spectral theory, The Chern Symposium 1979, edited by W.-Y. Hsiang et al. (Springer, New York, 1980).

${ }^{18}$ E. G. Kalnins, W. Miller, Jr., and G. J. Reid, "Separation of variables for complex Riemannian spaces of constant curvature 1. Orthogonal separable coordinates for coordinates $S_{n C}$ and $E_{n C}$," Proc. R. Soc. London, Ser. A 394, 183-206 (1984).

${ }^{19} \mathrm{~S}$. Wojciechowski, “Integrable one particle potentials related to the Neumann system and the Jacobi problem of geodesic motion on an ellipsoid," Phys. Lett. A 107, 106 (1985).

${ }^{20}$ S. Wojciechowski, “A one particle potential integrable on a family of quadrics,” Lett. Math. Phys. 9, 221 (1985). 